\title{
Nation-Wide Trends in Incidence-Based Mortality of Patients with Ocular Melanoma in USA: 2000 to 2018
}

\author{
Srijan Valasapalli id ${ }^{\prime}$ \\ Achuta Kumar Guddati iD ${ }^{2}$ \\ 'Department of Medicine, Carle \\ Foundation Hospital, Urbana, IL, 6I80I, \\ USA; ${ }^{2}$ Division of Hematology/Oncology, \\ Georgia Cancer Center, Augusta \\ University, Augusta, GA, 30909, USA
}

Background: Ocular and orbit melanoma is a rare subtype of melanoma for which outcomes have not been adequately reported. We have analyzed the incidence-based mortality trends of ocular and orbit melanoma over 15 years in USA. Most ocular melanomas originate from the uvea and, to a lesser extent, from the conjunctiva. Primary orbital melanoma is exceedingly rare. Methods: The Surveillance, Epidemiology, and End Results (SEER) database was queried to find the incidence-based mortality for all patients diagnosed with ocular and orbit melanoma for the years 2000 to 2018. Results were grouped by gender and race (Caucasian/White, African American/ Black, American Indian/Alaskan Native, and Asian/Pacific Islanders). A paired $t$-test was used to determine the statistically significant difference between various subgroups $(\mathrm{p}<0.05)$.

Results: Incidence-based mortality has been the highest in Caucasian/White patients from 2000 to 2018, followed by African American/Black and Asian/Pacific Islander patients. American Indian/Alaskan native patients appear to have the least mortality. There was a statistically significant difference $(\mathrm{p}<0.05)$ in mortality between Caucasian/White patients from 2000 to 2018, and African American/Black and Asian/Pacific Islander patients. The sample size for African American/Black and American Indian/Alaskan native patients was too low to discern a meaningful trend in mortality. Overall, it appears that Caucasian males and females have a far higher and worsening incidence-based mortality compared to other races.

Conclusion: Ocular melanoma and orbit melanoma are rare entities that are predominantly seen in Caucasian/White patients. This study shows that incidence-based mortality has been worsening for these patients in the past two decades. These entities have a poor prognosis and have not been studied extensively in immunotherapy trials. There is a need for new clinical trials to help improve mortality rates.

Keywords: ocular melanoma, mortality, trend, incidence

\section{Introduction}

Melanoma is the fifth most common cancer in men and women, with increasing incidence. Cutaneous melanomas comprise $1 \%$ of all skin cancers and $90 \%$ of all melanomas. ${ }^{1}$ Patients with a history of cutaneous melanoma are at increased risk for non-cutaneous melanomas, and they comprise $10 \%$ of all melanoma diagnoses. Non-cutaneous melanomas are more aggressive and associated with worse outcomes compared with cutaneous melanomas. ${ }^{2}$ As per the International Rare Cancer Initiative (ICRI), ocular melanoma is one of the rare forms of melanoma, comprising less than $5 \%$ of all melanomas. ${ }^{3}$ The melanomas mostly stem from melanocytes of the uveal tract $(>85-90 \%)^{4,5}$ followed by conjunctiva and orbit. The incidence ranges from 5 to 7 per million in Caucasians ${ }^{3,4,6}$ and $0.2-0.3 /$ million
Correspondence: Achuta Kumar Guddati Division of Hematology/Oncology, Georgia Cancer Center, Augusta University, Augusta, GA, 30909, USA

Tel + I 3/2-404-8928

Email aguddati@augusta.edu 
in African American \& Asian populations. ${ }^{7}$ About 2500 adults are diagnosed every year with ocular melanoma in the United States. This study investigates the incidencebased mortality in a gender and race-specific context in the United States.

Ocular melanomas most commonly arise from pigmented cells of the choroid ( $>90 \%$ ), followed by the ciliary body $(6 \%)$ and then the iris (4\%). While ciliary body melanomas carry the worst prognosis, the iris melanomas carry the best prognosis $(4 \%))^{5,8,9}$ The choroid, the ciliary body, and the iris are collectively called the uvea. Hence, Uveal Melanoma (UM) is an umbrella nomination. Most choroidal and ciliary body melanomas are thought to arise de novo, but some appear from pre-existing nevi. ${ }^{10}$ While in half the patients, this could be an incidental finding, the other half present with floaters, flashes, or visual field defects. Risk factors for developing ocular melanoma include male gender, ${ }^{11}>50$ years of age, ${ }^{12}$ Caucasian race, ${ }^{5,13}$ light-colored eyes, congenital melanocytosis, ${ }^{14}$ sensitivity to sunburn $(>50 \%$ cases seen in the Western US), ${ }^{15} \mathrm{UV}$ ray exposure, positive family history and germline mutations. ${ }^{4,10}$

Fundoscopy, ultrasonography, and fluorescein angiography are the primary modalities for diagnosis. Fine needle aspiration is now widely being performed for molecular testing and subsequent clinical trial enrollment, which can be valuable from a prognostic standpoint. ${ }^{5}$ Local therapies are efficacious in localized tumors; however, due to the extremely poor prognosis of metastatic ocular melanoma, more trials are warranted to determine optimal treatment. Different mutations identified thus far have acted as prognostic markers namely: Eukaryotic Translation Initiation Factor 1AX (EIF1AX), Splicing Factor 3B subunit 1 (SF3B1), BRCA associated protein 1 (BAP1), loss of nATM gene ${ }^{16}$ and most popularly Guanine Nucleotide Binding Proteins Q and 11 (GNAQ and GNA11) that lead to Mitogen-Activated Protein Kinase (MAPK) signaling pathways. ${ }^{17-19}$ While melanomas with mutations in E1F1AX and SF3B1 carry a relatively good prognosis, mutations in BAP1 have the worst prognosis. $30-50 \%$ of the UMs still metastasize mostly within 15-20 years and respond very poorly to chemotherapy. ${ }^{6,20}$ The most commonly used predictor to detect metastasis is monosomy $3 .^{10}$

The mortality rate of $50 \%$ is unchanged despite treatment advances in several decades, and the 5-year cancer-specific survival has been relatively stable without any decrease in the last few decades. ${ }^{4,21}$ High risk of metastasis is hypothesized secondary to micro-metastases, followed by a variable latency period prior to the emergence of overt metastatic disease. ${ }^{10}$ Managements mainly depends on the size, location of the tumor, extraocular extension, presence, or absence of metastasis. While there is no set management approach, the roles of various chemo and immunotherapy agents are being pursued to improve mortality rates. This study was presented in the form of an abstract at ASCO 2019.

\section{Methods \\ Data Source}

Data for the current study was extracted from the SEER database indicating the incidence-based mortality for all the patients diagnosed with ocular and orbital melanoma between the years 2004 and 2018 in USA. The SEER database is a registry of patients diagnosed with cancers in several geographical locations. It was established in 1971 under the National Cancer Act to maintain a registry of populationbased cancers. ${ }^{22}$ The SEER database is considered to be the most reliable source of incidence of cancers and survival. ${ }^{23}$

Cases of ocular melanoma and orbital melanoma were identified using International Classification of Disease for Oncology codes provided in the SEER database using the 18 county sunset detaining the incidence-based mortality. Variables such as gender and race, sub-grouped by races (Black/African-American, White/Caucasian, American Indian/Alaska natives, Asian/Pacific Islanders) were extracted using a session named: Eye and Orbit Melanoma 2000-2018. No patient consent was deemed to be necessary as there was no patient contact. Due to retrospective nature of the study and the use of de-identified patient data, the study was exempt from IRB review. Incidence-based mortality rate was defined as the number of deaths due to these cancers among the total number of patients that are diagnosed as evidenced in the SEER database.

\section{Statistical Analysis}

A paired $t$-test was used to determine statistical significance (defined as $\mathrm{P}$ value $<0.05$ ) between subgroups for incidence-based mortality. Linearized trend lines were used to compare mortality trends between males and females and across all four racial groups mentioned above.

\section{Results}

The overall incidence-based mortality rate was found using SEER analysis for the period of study, which is from the year 2000 to 2018. Incidence-based mortality for all patients diagnosed with ocular and orbit melanoma is depicted in Table 1. 
Table I Average Incidence-Based Mortality from 2000 to 2018 for Ocular Melanoma Across Race and Gender

\begin{tabular}{|l|c|c|}
\hline Race & $\begin{array}{c}\text { Incidence } \\
\text { Count }\end{array}$ & $\begin{array}{c}\sim \text { Incidence Rate per } \\
\text { Million of the } \\
\text { Population }\end{array}$ \\
\hline White & 3360 & 0.0025 \\
Male & 1841 & 0.0032 \\
Female & 1519 & 0.0002 \\
Black & 30 & 0.0002 \\
Male & 22 & 0.0004 \\
Female & 8 & 0.0001 \\
American Indian/Alaska & 12 & 0.0008 \\
Male & 5 & 0.0001 \\
Female & 7 & 0.0007 \\
Asian or Pacific Islander & 53 & 0.0004 \\
Male & 29 & 0.0005 \\
Female & 24 & 0.0003 \\
Unknown & 13 & $\sim$ \\
Male & 6 & $\sim$ \\
Female & 7 & $\sim$ \\
\hline
\end{tabular}

Note: Not Calculated.

The mortality was highest among the whites as compared to other races. The rate is higher in males compared to females in all the ethnic groups. A one-sample $t$-test was performed to determine the statistically significant difference between the incidence-based mortality among ocular and orbit melanomas in the four races shown in Table 1. Among the white population, this mortality rate is marginally higher, with a p-value $<0.05$. The trend of incidence-based mortality in Caucasian males and females from 2000 to 2018 is depicted in Figure 1. Incidence-based mortality in Whites was followed by Blacks/American
Africans, followed by Asians/Pacific islanders. American Indians/Alaska natives seem to have the least mortality. However, in light of the low sample size in Blacks/ Africans and American Indians/Alaska natives, it was hard to discern a meaningful trend. For the population designated as an unknown race, it was difficult to calculate incidence-based mortality rates due to low numbers among the population incidences.

\section{Discussion}

This study is a population-based analysis of trends in incidence-based mortality rates of ocular and orbital melanoma. The data of patients (including males and females) belonging to 4 ethnic groups, namely Black/African American, White/Caucasian, American Indian/Alaska natives, Asian/Pacific Islanders, were extracted from the SEER database from 2000 to 2018 . We included variables such as race and gender to evaluate mortality, which has shown a statistically significant value of high incidencebased mortality among White/Caucasians. Aronow et al have analyzed trends in incidence, treatment, and survival of UM cases in the US from 1973 to 2013. The ageadjusted incidences (with a mean of 5.2 per million) have been stable almost since 1973. They have shown an increased incidence in males compared to females and a statistically significant increase in the annual percentage of $0.5 \%$ in Whites. ${ }^{12}$ Singh et al studied the incidence of UM from 1973 to 1997 and found an age-adjusted incidence rate of 4.9 per million, also with a higher rate in males than females. The reasons for the increased predisposition in male gender is not clear but may be related to

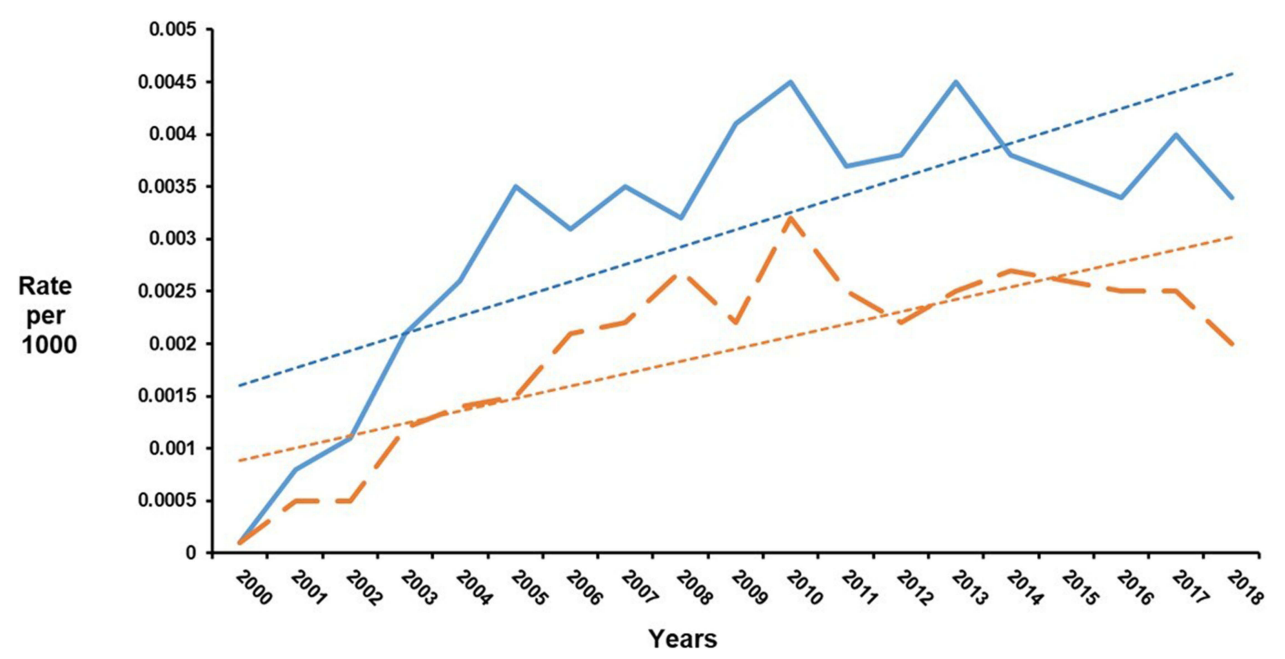

Figure I The trend of incidence-based mortality in Caucasian males and females from 2000 to 2018 . Dotted lines represent linearized trend lines. Solid blue line represents Caucasian males and orange dashed line represents Caucasian females. 
exposure to sun. With the enhancement in the diagnostic techniques, we see an elimination of "site-not-specified" subtypes. Lane et al compared a cohort of patients that were treated for metastasis versus those who were not. They also compared two cohorts followed in two different periods and have observed that there were similar survival rates, indicating the necessity of further research for effective treatments. ${ }^{24}$ Aronow et al also reported that 5-year relative survival remained unchanged over the past four decades despite evolving eye-sparing treatment. ${ }^{25}$ Ghazawi et al have performed a similar study comparing cases (from 1992 to 2010) in 2 Canadian provinces (British Columbia and Saskatchewan) and reported an incidence of 3.75 cases per million (52.1\% were males). They also noted a steady increase in the incidence of UM with an annual increase of 0.074 cases per million individuals per year. They noted a geographical difference. Incidence was higher in British Columbia, which is more western than Saskatchewan province. ${ }^{24}$

Most of the challenges in the determination of exact incidence rates also lie in the inclusion criteria in the studies for diagnostic criteria. The diagnosis of ocular melanomas in the past happened histo-pathologically postsurgical excision. More recently, imaging modalities such as ultrasonography, fluorescein angiography, and ocular echography have taken precedence over biopsies. ${ }^{4,26}$ However, FNA is being employed for molecular prognostic testing and clinical trial enrollment based on chromosome aberrations. ${ }^{27}$ Current treatment for non-metastatic UMs begins with close serial observation (tumors $<12 \mathrm{~mm}$ in diameter and $<2-3 \mathrm{~mm}$ in height). ${ }^{16}$ Radiation therapy (focal radiation, ocular brachytherapy, charged particle and photon-stereotactic) is also used most commonly as most uveal melanomas are radiosensitive. Transpupillary thermoplasty, laser photocoagulation, and photodynamic therapy are other non-invasive treatment modalities. Surgery (local resection, enucleation and exenteration for larger tumors) has not shown to improve any survival benefit over radiation. It is mainly reserved for patients in whom radiation therapy is unlikely to achieve adequate local tumor control due to extrascleral extension, risk of neovascular glaucoma, or susceptibility to unacceptable ocular radiation complications. $^{24,25,27,28}$

However, $30-50 \%$ of the Uveal Melanomas still metastasize and respond poorly to chemo/immunotherapy. ${ }^{6,29}$ Two-thirds of the metastases are to the liver, and the rest to the lung, bones, and skin. ${ }^{10}$ Prognosis of patients that developed metastasis is worse with an overall 1, and a 2-year survival rate of $13 \%$ and $8 \%$, respectively ${ }^{4}$ and there is a paucity in the current number of trials. Some studies have shown that metastasis would have started several years before the diagnosis of a primary tumor, which makes the study of mortality more challenging. ${ }^{30}$ The limitations are different pathogenesis and oncogenic drivers, the vast majority of them being GNAQ11. ${ }^{22}$ Therefore, targeting $G$ protein signaling in this cancer type is expected to be fruitful. ${ }^{31}$ Understanding of cytogenetics, gene expression profiling, and mutations of genes including but not limited to GNAQ11, BAP1, SF3B1 \& EIF1AX is crucial in laying the foundation for immunotherapy. There are very few clinical trials that are in the recruiting phase, and more are required. Immune checkpoint inhibitors like Pembrolizumab, Ipilimumab, Nivolumab, and Relatlimab are some of the agents in currently ongoing clinical trials. Najjar et al compared the efficacy of dual immune checkpoint inhibition, including nivolumab and ipilimumab (a monoclonal antibody that blocks CTLA-4). Although there is a better response compared to that of a single agent, the efficacy is lower than in metastatic cutaneous melanoma, and there are ongoing several clinical trials based on them. ${ }^{31,32}$ Rossi et al concluded that pembrolizumab had a remarkable disease control in patients that are responding. ${ }^{27}$ Targeted therapies, including Selumetinib (MAPK kinase inhibitor) plus dacarbazine as opposed to placebo plus dacarbazine, are currently under phase-3 double-blinded randomized controlled trials. Selumetinib has so far shown to improve PFS, although not OS. Zanella et al discuss the possibility of a synergistic effect of Pembrolizumab and Selumetinib via more trials as they individually have proven to improve PFS. ${ }^{33}$ Other trials targeting pathways like VEGF, KIT, Tyrosine kinase have not resulted in noteworthy responses. ${ }^{34}$ c-Met, a receptor tyrosine kinase, is also of interest and Crizotinib (a small molecule inhibitor selective for c-Met, anaplastic lymphoma kinase, and ROS1) has shown to be promising in mouse models which can be potentially extrapolated to humans via clinical trials. ${ }^{27}$ Interestingly, Luke JJ via in-vitro studies, has discovered the potential use of dihydropyridine calcium channel blockers like Amlodipine since calcium signaling pathway is known to be involved in cells with mutant GNAQ. They suggest further animal model studies for validation. ${ }^{30}$ Adjuvant therapies like hepatic artery chemoembolization in the treatment of hepatic metastasis have shown to be beneficial in patients, especially with the nodular metastatic pattern. ${ }^{29,35}$ 
The COMS (Collaborative Ocular Melanoma Study) medial melanoma trial and NHS study compare the mortality differences between the patients that elected treatment (brachytherapy/enucleation) vs those who have not elected treatment, respectively. The 5-year mortality for COMS was $18 \%$, while the 3 -year mortality for the NHS was $30 \%$. Although not statistically significant, adjustment to patient age and tumor size, the relative risk of death was $50 \%$ higher in NHS patients compared to COMS patients. ${ }^{36}$ Considering this a rare form of cancer with potential for discoveries of treatment, further research on methods to detect micrometastases and clinical trials involving immunotherapy is needed. Khoja et al also discuss setting a benchmark like six-month PFS and one-year OS to facilitate future trials. It gives a scope to consider the prognostic factors to target appropriate sub-groups, given the heterogeneity of disease outcomes. Currently, there is a lack of standard of care established benchmarks for comparison. Global collaboration to define staging and prognostication can serve as a better comparison between different ongoing therapies. ${ }^{37}$ UM Cure 2020 is an initiative in Europe that is dedicated to promoting more fundamental and clinical research to identify treatment options for uveal melanomas. ${ }^{38}$ Considering the immunogenic pathogenicity of other tumors like hypermutated cancers and lung cancers, uveal melanomas also necessitate the need for further basic science research detailing immunotherapy. ${ }^{39}$ The search for mutations listed above can help identify specific targets, and more clinical trials are warranted for the need of adjuvant therapies in high-risk cases. ${ }^{39}$ Limitations of SEER-based studies include, underreporting, selection bias, patient migration, coding reliability and missing data which are all typically inherent in an observational database.

\section{Conclusion}

This study illustrates that the incidence-based mortality trends in patients with ocular/orbit melanoma with race and gender stratification. Whites/Caucasians are shown to have the highest rate, followed by Blacks/African Americans, and these results are statistically significant (p-value $<0.05$ ). Males are shown to have higher rates compared to females among all the ethnic groups. The prognosis of patients developing metastatic ocular melanoma is very poor. Because this is a form of rare cancer/ orphan disease, a limited number of clinical trials have been conducted. Further multicenter trials are needed to fully understand the role of Immune Checkpoint Inhibitors for the treatment of uveal melanomas.

\section{Ethics Statement}

This study is based on a publicly available national database which has de-identified patient data with no patient contact and has been deemed to be exempt from IRB approval.

\section{Funding}

The authors declare that there was no funding for this.

\section{Disclosure}

The authors report no conflicts of interest in this work. The abstract was presented at ASCO 2019.

\section{References}

1. Chang AE, Karnell LH, Menck HR. The national cancer data base report on cutaneous and noncutaneous melanoma: a summary of 84,836 cases from the past decade. The American college of surgeons commission on cancer and the American cancer society. Cancer. 1998;83(8):1664-1678. doi:10.1002/(SICI)1097-0142(19981015) 83:8<1664::AID-CNCR23>3.0.CO;2-G

2. Beroukhim K, Pourang A, Eisen DB. Risk of second primary cutaneous and noncutaneous melanoma after cutaneous melanoma diagnosis: a population-based study. J Am Acad Dermatol. 2020;82 (3):683-689. doi:10.1016/j.jaad.2019.10.024

3. Boyd N, Dancey JE, Gilks CB, et al. Rare cancers: a sea of opportunity. Lancet Oncol. 2016;17(2):e52-e61. doi:10.1016/S14702045(15)00386-1

4. Mahendraraj K, Lau C, Lee I, et al. Trends in incidence, survival, and management of uveal melanoma: a population-based study of 7516 patients from the Surveillance, Epidemiology, and End Results database (1973-2012). Clin Ophthalmol. 2016;10:2113-2119. doi:10. 2147/OPTH.S113623

5. Kaliki S, Shields CL. Uveal melanoma: relatively rare but deadly cancer. Eye (Lond). 2017;31(2):241-257. doi:10.1038/eye.2016.275

6. Derrien AC, Rodrigues M, Eeckhoutte A, et al.. Germline MBD4 mutations and predisposition to uveal melanoma. J Natl Cancer Inst. 2020;113:80-87.

7. Thomsen H, Chattopadhyay S, Hoffmann P, et al. Genome-wide study on uveal melanoma patients finds association to DNA repair gene TDP1. Melanoma Res. 2020;30(2):166-172. doi:10.1097/CMR. 0000000000000641

8. OMF.Available from: http://www.ocularmelanoma.org/disease.htm. Accessed July 26, 2021.

9. Culp MB, Benard V, Dowling NF, et al. Ocular melanoma incidence rates and trends in the United States, 2001-2016. Eye (Lond. 2020;35:687-689.

10. Jager MJ.Uveal melanoma. Nat Rev Disease Primers. 2020;6(1):24.

11. Gass JD. Problems in the differential diagnosis of choroidal nevi and malignant melanomas. The XXXIII Edward Jackson Memorial Lecture. Am J Ophthalmol. 1977;83(3):299-323. doi:10.1016/00029394(77)90726-7

12. Singh AD, Turell ME, Topham AK. Uveal melanoma: trends in incidence, treatment, and survival. Ophthalmology. 2011;118 (9):1881-1885. doi:10.1016/j.ophtha.2011.01.040

13. Egan KM, Seddon JM, Glynn RJ, et al. Epidemiologic aspects of uveal melanoma. Surv Ophthalmol. 1988;32(4):239-251. doi:10.10 16/0039-6257(88)90173-7

14. Margo CE, Mulla Z, Billiris K. Incidence of surgically treated uveal melanoma by race and ethnicity. Ophthalmology. 1998;105 (6):1087-1090. doi:10.1016/S0161-6420(98)96012-2 
15. Singh AD, De Potter P, Fijal BA, et al. Lifetime prevalence of uveal melanoma in white patients with oculo(dermal) melanocytosis. Ophthalmology. 1998;105(1):195-198. doi:10.1016/S0161-6420(98) 92205-9

16. Aaberg TM, Cook R, Oelschlager K, et al. Current clinical practice: differential management of uveal melanoma in the era of molecular tumor analyses. Clin Ophthalmol. 2014;8:2449-2460. doi:10.2147/ OPTH.S70839

17. Jha J, Singh MK, Singh L, et al. Prognostic relevance of ATM protein in uveal melanoma and its association with clinicopathological factors. Int J Clin Oncol. 2019;24(12):1526-1535. doi:10.1007/ s10147-019-01519-6

18. Steeb T, Hayani KM, Förster P, et al. Guidelines for uveal melanoma: a critical appraisal of systematically identified guidelines using the AGREE II and AGREE-REX instrument. J Cancer Res Clin Oncol. 2020;146(4):1079-1088. doi:10.1007/s00432-020-03141-w

19. Singh AD. Prognostication of uveal melanoma: a work in progress. JAMA Ophthalmol. 2016;134(7):740-741. doi:10.1001/jamaophthalmol.2016. 1070

20. Drabarek W, Yavuzyigitoglu S, Obulkasim A, et al. Multi-modality analysis improves survival prediction in enucleated uveal melanoma patients. Invest Ophthalmol Vis Sci. 2019;60(10):3595-3605. doi:10.1167/iovs. 18-24818

21. Sandinha MT, Farquharson MA, McKay IC, et al. Monosomy 3 predicts death but not time until death in choroidal melanoma. Invest Ophthalmol Vis Sci. 2005;46(10):3497-3501. doi:10.1167/ iovs.05-0613

22. Eskelin S, Pyrhönen S, Summanen P, et al. Tumor doubling times in metastatic malignant melanoma of the uvea: tumor progression before and after treatment. Ophthalmology. 2000;107(8):1443-1449. doi:10.1016/S0161-6420(00)00182-2

23. Singh AD, Topham A. Incidence of uveal melanoma in the United States: 1973-1997. Ophthalmology. 2003;110(5):956-961. doi:10.10 16/S0161-6420(03)00078-2

24. Aronow ME, Topham AK, Singh AD. Uveal melanoma: 5-year update on incidence, treatment, and survival (SEER 1973-2013). Ocul Oncol Pathol. 2018;4(3):145-151. doi:10.1159/000480640

25. Lane AM, Egan KM, Kim IK, et al. Mortality after diagnosis of small melanocytic lesions of the choroid. Arch Ophthalmol. 2010;128 (8):996-1000. doi:10.1001/archophthalmol.2010.166

26. Ghazawi FM, Darwich R, Le M, et al. Uveal melanoma incidence trends in Canada: a national comprehensive population-based study. Br J Ophthalmol. 2019;103(12):1872-1876.

27. Chattopadhyay C, Kim DW, Gombos DS, et al. Uveal melanoma: from diagnosis to treatment and the science in between. Cancer. 2016;122(15):2299-2312. doi:10.1002/cncr.29727
28. Group COMS. The COMS randomized trial of iodine 125 brachytherapy for choroidal melanoma: v. Twelve-year mortality rates and prognostic factors: COMS report No. 28. Arch Ophthalmol. 2006;124(12):1684-1693.

29. Souto EB, Zielinska A, Luis M, et al. Uveal melanoma: physiopathology and new in situ-specific therapies. Cancer Chemother Pharmacol. 2019;84(1):15-32. doi:10.1007/s00280019-03860-z

30. Surriga O, Rajasekhar VK, Ambrosini G, et al. Crizotinib, a c-Met inhibitor, prevents metastasis in a metastatic uveal melanoma model. Mol Cancer Ther. 2013;12(12):2817-2826. doi:10.1158/1535-7163. MCT-13-0499

31. Najjar YG, Navrazhina K, Ding F, et al. Ipilimumab plus nivolumab for patients with metastatic uveal melanoma: a multicenter, retrospective study. J Immunother Cancer. 2020;8(1):e00331. doi:10.1136/jitc-2019-000331

32. Larribère L, Utikal J. Update on GNA alterations in cancer: Implications for uveal melanoma treatmen. Cancers (Basel). 2020;12(6):1524.

33. Rossi E, Pagliara MM, Orteschi D, et al. Pembrolizumab as first-line treatment for metastatic uveal melanoma. Cancer Immunol Immunother. 2019;68(7):1179-1185. doi:10.1007/s00262-019-023 $52-6$

34. Zanella A, Doussot A, Puzenat E, et al. MEK inhibitors combined with programmed cell death-1 blockade immunotherapy for metastatic uveal melanoma: is it warranted? Melanoma Res. 2020;30 (6):606-607. doi:10.1097/CMR.0000000000000677

35. Luke JJ. The newest treatments for uveal melanoma. Clin Adv Hematol Oncol. 2019;17(9):490-493.

36. Dayani PN, Gould JE, Brown DB, et al. Hepatic metastasis from uveal melanoma: angiographic pattern predictive of survival after hepatic arterial chemoembolization. Arch Ophthalmol. 2009;127 (5):628-632. doi:10.1001/archophthalmol.2009.45

37. Straatsma BR. Mortality after deferral of treatment or no treatment for choroidal melanoma. Indian $J$ Ophthalmol. 2018;66 (10):1395-1400. doi:10.4103/ijo.IJO_1499_18

38. Khoja L, Atenafu EG, Suciu S, et al. Meta-analysis in metastatic uveal melanoma to determine progression free and overall survival benchmarks: an international rare cancers initiative (IRCI) ocular melanoma study. Ann Oncol. 2019;30(8):1370-1380. doi:10.1093/ annonc/mdz176

39. Rodrigues M, de Koning L, Coupland S, et al. So close, yet so far: discrepancies between uveal and other melanomas. a position paper from UM cure 2020. Cancers (Basel). 2019;11(7):1032. doi:10.3390/ cancers 11071032
International Journal of General Medicine

\section{Publish your work in this journal}

The International Journal of General Medicine is an international, peer-reviewed open-access journal that focuses on general and internal medicine, pathogenesis, epidemiology, diagnosis, monitoring and treatment protocols. The journal is characterized by the rapid reporting of reviews, original research and clinical studies across all disease areas. The manuscript management system is completely online and includes a very quick and fair peer-review system, which is all easy to use. Visit http://www.dovepress.com/ testimonials.php to read real quotes from published authors. 